\title{
Equações segmentadas de perfil do tronco geradas a partir da equação que descreve sólidos geométricos
}

\author{
Valdir Carlos Lima de Andrade ${ }^{1}$ Agostinho Lopes de Souza², Natalino Calegário ${ }^{3}$ \\ 1 Universidade Federal do Tocantins, Curso de Engenharia Florestal, CEP 77.402-970, Gurupi, TO, Brasil \\ 2Universidade Federal de Viçosa, Departamento de Engenharia Florestal, CEP 36.570-000, Viçosa, MG, Brasil \\ ${ }^{3}$ Universidade Federal de Lavras, Departamento de Ciências Florestais, CEP 37.200-000, Lavras, MG, Brasil
}

*Autor correspondente:

vclandrade@hotmail.com

Termos para indexação:

Estatística experimental

Dendrometria

Cubagem rigorosa

Index terms:

Experimental Statistics

Dendrometry

Accurate Scaling

Histórico do artigo:

Recebido em 27/01/2014

Aprovado em 11/08/2014

Publicado em 30/12/2014

doi: $10.4336 / 2014 . p f b .34 .80 .654$

\begin{abstract}
Resumo - Neste trabalho, a partir da equação: $y=b x^{r}$, desenvolveu-se uma metodologia que vincula os diferentes sólidos geométricos em quatro segmentos do tronco de árvores em pé. Foram utilizados dados de 1.297 árvores cubadas do híbrido entre Eucalyptus urophylla e Eucalyptus grandis para análises preliminares e dados independentes de outras 65 árvores cubadas para validação da metodologia desenvolvida quanto à predição do perfil do tronco e dos volumes. Os critérios de avaliação da qualidade e adequação da metodologia basearam-se na análise gráfica dos desvios e estatísticas de desvio percentual médio, erro padrão residual, exatidão obtida pelo teste de quiquadrado e análise de variância por meio do delineamento inteiramente casualizado em parcelas subdivididas com aplicação do teste de Dunnett, ambos ao nível de 0,05 de significância. Concluiu-se que a partir de um sólido geométrico, ou protótipo dendrométrico otimizado, é possível simular a cubagem de árvores em pé, medindo-se apenas os diâmetros do tronco situados em: 0,3 m, 1,3 m, h-2/2, h-1,25/1,25 e a altura total $\mathrm{h}$, cujos procedimentos foram incluídos em uma metodologia denominada método da altura relativa com dois diâmetros $(\mathrm{hr} 2 \mathrm{D})$.
\end{abstract}

\section{Taper segmented equation generated from the equation which describes geometric solids}

\begin{abstract}
In this work, from the equation $y=b x^{r}$, a methodology that links the different geometric solids in four segments of standing trees trunks was developed. Data from 1297 scaled trees of the hybrid Eucalyptus grandis and Eucalyptus urophylla were used for the preliminary analysis and independent data from another 65 scaled trees for the validation of the developed methodology related to the taper and volume predictions. The evaluation criteria of the quality and fitness of the methodology were based on the graphic analyses of the residuals and on the followings statistics: mean percentual deviation, residual standard error, accuracy obtained from the Chi-square test and the variance analyses by the Entirely Randomized Design in Subdivided Blocs with the use of the Dunnett test, both at 0.05 significance level. It was concluded that from a greometric solid, or optimized dendrometric prototype, it is possible to simulated the scaling of standing trees, measuring the trunk diameters located only at: $0.3 \mathrm{~m}, 1.3 \mathrm{~m}, \mathrm{~h}-2 / 2$ and on $\mathrm{h}-1,25 / 1,25$, besides the total height $\mathrm{h}$, whose processes were included in a methodology named Relative Height Method with Two Diameters (hr2D).
\end{abstract}




\section{Introdução}

$\mathrm{O}$ uso de informações volumétricas com um alto nível de confiabilidade, é fundamental para a tomada de decisão, otimização e uso sustentável da madeira produzida nos povoamentos florestais. Essa informação torna-se ainda mais importante quando se comercializa diferentes sortimentos. É importante, então, atentar para o nível de representatividade e acurácia das equações para multiprodutos da madeira que serão geradas, a partir de dados de cubagem de árvores.

Uma alternativa para minimizar o vício no inventário, advindo de equações de multiprodutos, é cubar árvores selecionadas próximas ou dentro das parcelas, similar ao que foi realizado por Couto \& Vetorazzo (1999) e Chichorro (2000). Tal procedimento, de se amostrar árvores próxima das parcelas de inventário, além de tornar compatível as equações de multiprodutos e de árvores amostradas, proporciona melhor nível de representatividade nas árvores cubadas devido à distribuição das parcelas na área inventariada, pois a relação volumétrica é influenciada pelo local, idade, posição da árvore e pela dependência entre indivíduos.

É importante, então, conduzir um inventário florestal empregando-se metodologias que permitam cubar árvores de forma simultânea às medições das parcelas e que envolvam menor quantidade de diâmetros medidos no tronco, para reduzir custos e dificuldades técnicas-operacionais. O método da altura relativa é indicado nesse caso, porque exclui a cubagem rigorosa de árvores e permite simulá-la a partir da medição de diâmetros do tronco situados em $0,3 \mathrm{~m}$ e em (h-2)/2 m com a árvore em pé, considerando também a $1,3 \mathrm{~m}$ e a altura total (h), conforme feito por Leite \& Andrade (2002).

$\mathrm{Na}$ realidade, para gerar equações de perfil do tronco empregando o método da altura relativa, é necessário medir apenas o diâmetro em (h-2)/2, pois em 1,3 m a medição é feita obrigatoriamente em qualquer inventário florestal e, em $0,3 \mathrm{~m}$, por ser próximo do solo, tem a sua medição facilitada. Além disso, na maioria dos plantios florestais, as árvores tem diâmetro igual a zero em h, o que torna dificultosa a sua medição apenas em (h-2)/2 por ser feita na árvore em pé (Andrade, 2006). No entanto, como existem instrumentos próprios para a medição de diâmetros do tronco de árvores em pé, como: Relascópio de Bitterlich, Pentaprisma de Weller e Criterion, o problema para obter o diâmetro em (h-2)/2, com árvores-amostra em pé, é solucionado pelo emprego de tais instrumentos.

Diante do que foi exposto e considerando que o método da altura relativa se baseia no sólido geométrico cone, utilizando-se troncos de cone em segmentos do tronco de árvores em pé para descrever o seu perfil longitudinal, conforme demonstrado por Andrade (2006), conduziu-se este estudo tendo como objetivo avaliar equações de perfil do tronco geradas a partir de outros sólidos geométricos atrelados à medição de poucos diâmetros do tronco de árvores em pé.

\section{Material e métodos}

Este estudo utilizou dados de cubagem de 1.362 árvores-amostra do híbrido entre Eucalyptus grandis e Eucalyptus urophylla, cujas árvores foram cortadas em plantios comerciais localizadas na Região Nordeste do Estado da Bahia, com idades entre 5 e 7 anos e tendo espaçamento de plantio de 3,0 m X 1,5 m. Os dados da cubagem foram obtidos pela medição dos diâmetros nas posições de $0,1 \mathrm{~m}, 0,3 \mathrm{~m}, 0,7 \mathrm{~m}, 1,3 \mathrm{~m}, 2 \mathrm{~m}$ e a cada metro até o diâmetro mínimo de $1 \mathrm{~cm}$ com casca.

Após obter os dados de cubagem, estes foram utilizados na definição do protótipo dendrométrico (PD) que, em média, minimiza o desvio entre diâmetros deste PD e diâmetros reais ao longo do tronco de árvores, denominado de: protótipo dendrométrico otimizado (PDO). A equação do PDO foi considerada como sendo uma equação de perfil do tronco gerada, separadamente, para quatro segmentos do tronco de árvores, quais sejam:

- Porção basal do tronco (PBT) definido entre $0,0 \mathrm{~m}$ a $1,3 \mathrm{~m}$;

- Porção mediana do tronco (PMT) definida entre 1,3 m a (h-1,4)/1,4;

- Porção superior do tronco (PST) definido entre (h-1,4)/1,4 a (h-1,1)/1,1; e

- Porção apical do tronco (PAT) definido entre (h-1,1)/1,1 a h.

$\mathrm{Na}$ identificação do PDO, seguiram-se os procedimentos apresentados em Andrade (2006) e em Andrade et al. (2009), que se iniciaram com a equação (1): $y=b x^{r}$. Nesta, conforme Gomes (1957), $y$ se 
refere ao raio da base de um sólido geométrico (SG), $x$ é o comprimento total de um SG, $r$ é o índice que descreve a forma de um SG (denominado neste trabalho de expoente-forma) e $b$ é uma constante que representa a taxa de afilamento.

Ao variar o expoente-forma da equação 1 , assumindo valores iguais à: $0,1 / 3,1 / 2,2 / 3,1$ e $3 / 2$, respectivamente, obtém-se: um cilindro, um paraboloide cúbico, um paraboloide quadrático, um paraboloide semi-cúbico, um conóide e um neilóide, conforme apresentado e ilustrado em Gomes (1957). Estes SG's são os que melhor se adéquam à forma de diferentes partes ao longo do tronco da grande maioria das árvores e são definidos como protótipos dendrométricos.

$\mathrm{O}$ uso de diferentes protótipos dendrométricos, para caracterizar o perfil do tronco de árvores, exigiu adaptações da equação 1 quanto aos limites fixos no tronco de árvores onde o diâmetro é assumido como o diâmetro de um determinado protótipo dendrométrico (PD), definidos como limite inferior hb e limite superior hv (Figura 1). Nesta situação, y da equação 1 passa a ser o raio em $\mathrm{hb}(\mathrm{db} / 2)$ e x passa a ser o comprimento do PD (hv-hb), virtualmente, inserido no tronco de árvores. Após as devidas transformações algébricas, o resultado é a equação 2.

Ao aplicar a equação 2 em 1 para substituir b, considerou-se $\mathrm{y}_{\mathrm{i}}$ sendo diferentes raios em $\mathrm{x}_{\mathrm{i} \text {-ésimas }}$ partes ao longo de um determinado PD. Obteve-se a equação 3 .

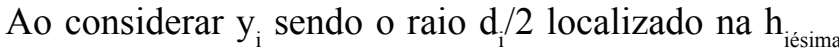
altura no tronco das árvores, sendo $x_{i}=h v-h_{i}$, onde hv está distante $1 \mathrm{~m}$ desta $\mathrm{h}_{\text {iésima }}$ altura no tronco de árvores, desejada para se saber $d_{i}$ (Figura 2), então, conforme Andrade (2006), o resultado são as equações 4 e 5.

As equações 4 e 5 caracterizam o perfil do tronco de árvores quando, em média, os diâmetros de parte do tronco destas sejam iguais aos diâmetros de parte de um determinado PD, sendo utilizado um tronco de PD para descrever um determinado segmento do tronco de árvores. Isto exige assumir um determinado nível de acurácia em relação ao perfil real, o que impõe às equações 4 e 5 o modelo das equações 6 e 7 , respectivamente, onde $\varepsilon$ é o erro ou resíduo.

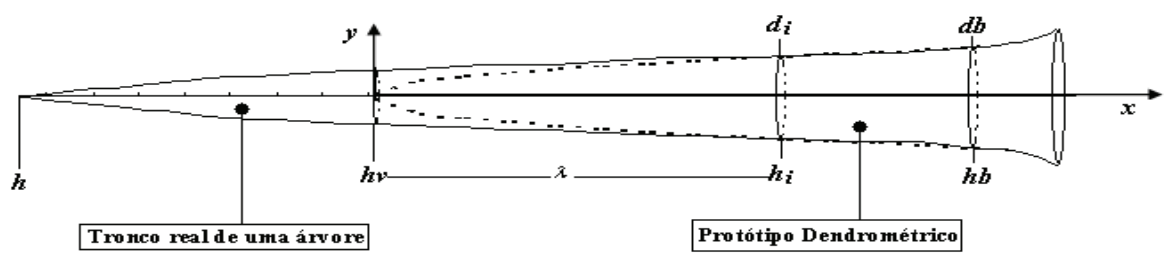

Figura 1. Tronco real de uma árvore, tendo um protótipo dendrométrico, fixado virtualmente entre os limites hv e hb. Em que: $\mathrm{y}=$ raio ao longo de um determinado $\mathrm{PD} ; \mathrm{x}=$ comprimento do $\mathrm{PD}$; hv = vértice do $\mathrm{PD}$; hb = base do PD coincidente com o tronco da árvore; $\mathrm{db}=$ diâmetro do tronco em hb assumido como diâmetro do PD; di = diâmetro do tronco da árvore; $\mathrm{hi}=$ altura referente à di; $1=$ ponta do $\mathrm{PD} ; \mathrm{h}=$ altura ou comprimento total da árvore.

$$
\begin{gathered}
b=\left(\frac{d b}{2}\right) /(h v-h b)^{r} \\
y_{i}= \pm\left[(d b / 2) /(h v-h b)^{r}\right] x_{i}^{r} \\
\hat{d}_{i}=d b\left[\left(h v-h_{i}\right) /(h v-h b)\right]^{r} \\
d_{i}=d b\left[(\lambda) /\left(h_{i}+\lambda-h b\right)\right]^{r} \\
d_{i}=d b\left[\left(h v-h_{i}\right) /(h v-h b)\right]^{r}+\varepsilon \\
d_{i}=d b\left[(\lambda) /\left(h_{i}+\lambda-h b\right)\right]^{r}+\varepsilon
\end{gathered}
$$


A análise dos resíduos obtidos com as equações 6 e 7, conforme Andrade (2006), permite definir o PD que, em média, pode ter parte de seus diâmetros assumidos como parte do tronco de árvores. Desta forma, as equações $6 \mathrm{e}$ 7, respectivamente, resultam nas equações de perfil do tronco 8 e 9 , onde $h_{\mathrm{i}}$ é a i-ésima altura no tronco de árvores e $d_{i}$ é o diâmetro estimado, ou, predito em $h_{\mathrm{i}}$ :

$$
\begin{aligned}
& d_{i}=d b\left[\left(h v-h_{i}\right) /(h v-h b)\right]^{r} \\
& \hat{d}_{i}=d b\left[(\lambda) /\left(h_{i}+\lambda-h b\right)\right]^{r}
\end{aligned}
$$

Considerando-se as porções do tronco PBT, PMT, PST e PAT, foram obtidas equações de PD que, em média, podem ter seus diâmetros assumidos como diâmetros do tronco em cada um destes segmentos. Assim, para um melhor entendimento de como se gerou as equações de perfil do tronco, recomenda-se recorrer à Andrade (2006), onde são apresentados todos os detalhes de como se chegou a tais equações de perfil do tronco.

Diante disso, para o desenvolvimento de uma equação de perfil do tronco na PBT, a partir de um protótipo dendrométrico otimizado (PDO), inicialmente, foram adotadas as equações 10 e 11 :

$$
\begin{gathered}
h v=\varphi_{1} d_{1,3} \\
\lambda=1,3+\exp \left(\varphi_{2} d_{1,3}\right)
\end{gathered}
$$

Em todas as deduções e transformações algébricas, respectivamente, feitas a partir da substituição de hv e $\lambda$ em 8 e 9 por 10 e 11, obteve-se as seguintes equações de perfil do tronco:

$$
\begin{gathered}
\hat{d}_{\left(0,0 \leq h_{i} \leq 1,3\right)}=d_{1,3}\left(\frac{1,3+\exp \left(\varphi_{2} c d_{1,3}\right)}{h_{i}+\exp \left(\varphi_{2} c d_{1,3}\right)}\right)^{r c} \\
\hat{d}_{\left(0,0 \leq h_{i} \leq 1,3\right)}=\left(\frac{d_{1,3}}{2}\right)\left[\left(\frac{\varphi_{1} c d_{1,3}-h_{i}}{\varphi_{1} c d_{1,3}-1,3}\right)^{r m b}+\left(\frac{1,3+\exp \left(\varphi_{2} c d_{1,3}\right)}{h_{i}+\exp \left(\varphi_{2} c d_{1,3}\right)}\right)^{r m b}\right] \\
\hat{d}_{\left(1,3<h_{i} \leq h r 1\right)}=\left(\frac{d_{1,3}}{2}\right)\left\{\left[\frac{(h-X m) / X m-h_{i}}{(h-X m) / X m-1,3}\right]^{r m a}+\left(\frac{\exp \left(\varphi_{2} c m d_{1,3}\right)}{h_{i}+\exp \left(\varphi_{2} c m d_{1,3}\right)-1,3}\right)^{r m a}\right\}
\end{gathered}
$$

Em que: $\hat{\mathrm{d}}\left(0,0 \leq \mathrm{h}_{\mathrm{i}} \leq 1,3\right)=$ diâmetro estimado/predito entre 0,0 à $1,3 \mathrm{~m}, \phi_{1}$ e $\phi_{2}, \phi_{1} \mathrm{c}, \phi_{2} \mathrm{c}, \phi_{1} \mathrm{cn}$ e $\phi_{2} \mathrm{cn}=$ parâmetros para serem calculados conforme Andrade (2006), $d_{1,3}=$ diâmetro medido a 1,3 $\mathrm{m}$ do terreno, rc, rmb e rma = expoentes-forma de um determinado PD à serem obtidos conforme detalhes apresentados em Andrade (2006), exp = inverso do logaritmo neperiano e os demais parâmetros já definidos anteriormente.

Por outro lado, no desenvolvimento de uma equação de perfil do tronco na PMT, inicialmente, foram adotadas as equações 15 e 16 :

$$
\begin{aligned}
& h v=(h-X) / X \\
& \lambda=\exp \left(\varphi_{2} d_{1,3}\right)
\end{aligned}
$$


Após as deduções e transformações algébricas, respectivamente, feitas a partir da substituição de hv e $\lambda$ em 8 e 9 por 15 e 16, obteve-se as seguintes equações de perfil do tronco:

$$
\begin{gathered}
\hat{d}_{\left(1,3<h_{i} \leq h r 1\right)}=d_{1,3}\left[\frac{(h-X) / X-h_{i}}{(h-X) / X-1,3}\right]^{r c a} \\
\hat{d}_{\left(1,3<h_{i} \leq h r 1\right)}=d_{1,3}\left[\frac{(h-X a) / X a-h_{i}}{(h-X a) / X a-1,3}\right]^{r c} \\
\hat{d}_{\left(1,3<h_{i} \leq h r 1\right)}=\left(\frac{d_{1,3}}{2}\right)\left\{\left[\frac{(h-X m) / X m-h_{i}}{(h-X m) / X m-1,3}\right]^{r m a}+\left(\frac{\exp \left(\varphi_{2} c m d_{1,3}\right)}{h_{i}+\exp \left(\varphi_{2} c m d_{1,3}\right)-1,3}\right)^{r m a}\right\}
\end{gathered}
$$

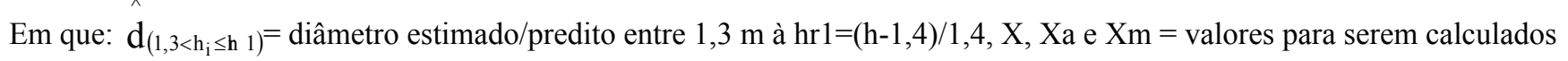
conforme Andrade (2006), $\mathrm{d}_{1,3}=$ diâmetro medido a 1,3 $\mathrm{m}$ do terreno, $\mathrm{rca}=$ expoente-forma de um determinado PD à ser obtido conforme detalhes apresentados em Andrade (2006). Os demais parâmetros já definidos anteriormente.

No desenvolvimento das equações de perfil do tronco na PST, similar ao que foi feito na PMT, obteve-se as seguintes equações de perfil do tronco:

$$
\begin{gathered}
\hat{d}_{\left(h r 1<h_{i} \leq h r 2\right)}=d r 1\left[\frac{(h-X) / X-h_{i}}{(h-X) / X-h r 1}\right]^{r c a} \\
\hat{d}_{\left(h r 1<h_{i} \leq h r 2\right)}=d r 1\left[\frac{(h-X a) / X a-h_{i}}{(h-X a) / X a-h r 1}\right]^{r c} \\
\hat{d}_{\left(h r 1<h_{i} \leq h r 2\right)}=\left(\frac{d r 1}{2}\right)\left\{\left[\frac{(h-X m) / X m-h_{i}}{(h-X m) / X m-h r 1}\right]^{r m a}+\left(\frac{\exp \left(\varphi_{2} c m d_{1,3}\right)}{h_{i}+\exp \left(\varphi_{2} c m d_{1,3}\right)-h r 1}\right)^{r m a}\right\}
\end{gathered}
$$

Em que: $\hat{\mathrm{d}}_{\left(\mathrm{h} 1<\mathrm{h}_{\mathrm{i}} \leq \mathrm{h} 2\right)}=$ diâmetro estimado/predito entre $\mathrm{hr} 1=(\mathrm{h}-1,4) / 1,4$ à $\mathrm{hr} 2=(\mathrm{h}-1,1) / 1,1$, dr1 $=$ diâmetro obtido em $\mathrm{hr} 1=(\mathrm{h}-1,4) / 1,4$. Os demais parâmetros já definidos anteriormente. 
Por fim, o que delimita a PAT no método da altura relativa deve levar em conta que, quando a $\mathrm{h}_{\mathrm{i} \text {-śsima }}$ altura no tronco é igual a $\mathrm{h}$ tem-se que $\mathrm{d}_{\mathrm{i}}$ é igual a zero. Portanto, para descrever o perfil do tronco na PAT, deve-se utilizar apenas a equação 8 porque hv tem que ser igual a h. Assim, ao fazer db igual a dr2, hb é hr2 e hv é h, obteve-se a seguinte equação de perfil do tronco:

$$
\hat{d}_{\left(h r 2<h_{i} \leq h\right)}=d r 2\left[\left(h-h_{i}\right) /(h-h r 2)\right]^{r a}
$$

Em que: $\hat{d}_{\left(h_{2}<h_{i} \leq h\right)}$ é o diâmetro na $h_{\text {i-ésima }}$ altura no tronco entre $h r 2=(h-1,1) / 1,1$ à $h, d r 2=$ diâmetro obtido em $\mathrm{hr} 2=(\mathrm{h}-1,4) / 1,4, \mathrm{ra}=$ expoente-forma de um determinado PD à ser obtido conforme detalhes apresentados em Andrade (2006). Os demais parâmetros já definidos anteriormente.

Diante destas considerações, foram avaliadas três possibilidades para se aplicar as equações de 12 à 23 para descrever o perfil do tronco de árvores em pé, quais sejam:

- Protótipo dendrométrico otimizado, obtido sem a medição de dois diâmetros do tronco (PDOSM2D);

- Protótipo dendrométrico otimizado, obtido com a medição de dois diâmetros do tronco (PDOCM2D); e

- Protótipo dendrométrico otimizado, obtido com a medição de dois diâmetros do tronco usando o vértice hv deste, virtualmente, inserido no tronco de árvores (PDOCM2Dhv).

Na possibilidade de uso do PDOSM2D, são empregadas as equações de perfil do tronco:

- uso de uma equação dentre 12,13 e 14 ,

- uso de uma equação dentre 17,18 e 19 ,

- uso de uma equação dentre 20,21 e 22 e

- uso da equação 23.

Na possibilidade de uso do PDOCM2D, devese obter o PDO a partir de diâmetros medidos em: $\mathrm{hra}=(\mathrm{h}-\mathrm{Xa}) / \mathrm{Xa}(24)$ e hrb $=(\mathrm{h}-\mathrm{Xb}) / \mathrm{Xb}$ ( 25 ), sendo $\mathrm{hr}_{\mathrm{a}}$ e $\mathrm{hr}_{\mathrm{b}}$ posições para se medir os diâmetros do tronco de árvores. Assim, para se obter o PDO próprio de cada porção do tronco de árvores em pé, são empregadas as mesmas equações do PDOSM2D, porém, obtendo-se o expoente-forma por meio de:

$$
\begin{aligned}
& r c_{i j} 0,3=\left[\operatorname{Ln}\left(d r_{0,3_{i j}}\right)-\operatorname{Ln}\left(d b_{j}\right)\right] /\left[\operatorname{Ln}\left(h v_{j}-0,3\right)-\operatorname{Ln}\left(h v_{j}-h b_{j}\right)\right] \\
& r c_{i j} a=\left[\operatorname{Ln}\left(d r_{a_{i j}}\right)-\operatorname{Ln}\left(d b_{j}\right)\right] /\left[\operatorname{Ln}\left(h v_{j}-h r_{a_{i j}}\right)-\operatorname{Ln}\left(h v_{j}-h b_{j}\right)\right] \\
& r c_{i j} b=\left[\operatorname{Ln}\left(d r_{b_{i j}}\right)-\operatorname{Ln}\left(d b_{j}\right)\right] /\left[\operatorname{Ln}\left(h v_{j}-h r_{b_{i j}}\right)-\operatorname{Ln}\left(h v_{j}-h b_{j}\right)\right]
\end{aligned}
$$

Onde $\mathrm{rc}_{\mathrm{ij}} 0,3$ é o expoente-forma que representa um PDO na i-ésima árvore da j-ésima classe de $\mathrm{d}_{1,3}$, usando o intervalo entre $\mathrm{hb}$ e $0,3 \mathrm{~m} ; \mathrm{rc}_{\mathrm{ij}}$ é o expoente-forma que representa um PDO na i-ésima árvore da j-ésima classe de $\mathrm{d}_{1,3}$, usando o intervalo entre hb e hra; $\mathrm{rc}_{\mathrm{ij}}$ be o expoente-forma que representa um PDO na i-ésima árvore da j-ésima classe de $\mathrm{d}_{1,3}$, usando o intervalo entre hb e hrb; dr ${ }_{0,3 i j}$ é o diâmetro medido em $0,3 \mathrm{~m}$; dra $\mathrm{ij}_{\mathrm{ij}}$ é o diâmetro medido em $\mathrm{hra}_{\mathrm{ij}}$; e drb $\mathrm{ij}_{\mathrm{ij}}$ é o diâmetro medido em hrb $\mathrm{ij}_{\mathrm{j}}$.

Na possibilidade de uso do PDOCM2Dhv, é acrescentado um $\theta_{1}$ ao vértice hv do PDO, de modo que se minimize o desvio entre diâmetros de parte deste PDO e de parte do tronco de árvores. Para isto, a partir da equação 8, obteve-se as seguintes equações de perfil do tronco: 


$$
\begin{aligned}
& \hat{d}_{i}=d_{1,3}\left[\frac{h v_{0,3}+\theta_{1} \text { ótimo }^{*}-h_{i}}{h v_{0,3}+\theta_{1} \text { ótimo }^{*}-1,3}\right]^{r_{\text {otimo }}} \\
& \hat{d}_{i}=d_{1,3}\left[\frac{h v a+\theta_{1} \text { ótimo }^{*}-h_{i}}{h v a+\theta_{1} \text { ótimo }^{*}-1,3}\right]^{r_{\text {otimo }}} \\
& \hat{d}_{i}=d_{1,3}\left[\frac{h v b+\theta_{1} \text { ótimo }^{*}-h_{i}}{h v b+\theta_{1} \text { ótimo }^{*}-1,3}\right]^{r_{\text {otimo }}}
\end{aligned}
$$

Onde:

$$
\begin{aligned}
& h v_{0,3}=\left[0,3-h b\left(d r_{0,3} / d b\right)^{(1 / r)}\right] /\left[1-\left(d r_{0,3} / d b\right)^{\left(\frac{1}{r}\right)}\right], \\
& h v a=\left[h r a-h b(d r a / d b)^{\left(\frac{1}{r}\right)}\right] /\left[1-(d r a / d b)^{\left(\frac{1}{r}\right)}\right] \mathrm{e} \\
& h v b=\left[h r b-h b(d r b / d b)^{\left(\frac{1}{r}\right)}\right] /\left[1-(d r b / d b)^{\left(\frac{1}{r}\right)}\right] .
\end{aligned}
$$

As três posibilidades de se usar um PDO para descrever o perfil do tronco de árvores em pé, inicialmente, foram avaliadas em cada porção do tronco de 1.297 árvores, juntamente com o método da altura relativa (AR), cuja metodologia emprega as seguintes equações segmentadas de perfil do tronco:

$$
\begin{aligned}
& \hat{\mathrm{d}}_{\left(0,0 \leq \mathrm{h}_{\mathrm{i}} \leq 1,3\right)}=\frac{2 \mathrm{~h}_{\mathrm{i}}-2,6}{\mathrm{CAR}_{\mathrm{I}}}+\mathrm{d}_{1,3}(32), \\
& \hat{\mathrm{d}}_{\left(1,3<\mathrm{h}_{\mathrm{i}} \leq \mathrm{hr} 1\right)}=\frac{2 \cdot \mathrm{h}_{\mathrm{i}}-2,6}{\mathrm{CAR}_{\mathrm{II}}}+\mathrm{d}_{1,3}(33), \\
& \hat{\mathrm{d}}_{\left(\mathrm{hr} 1<\mathrm{h}_{\mathrm{i}} \leq \mathrm{hr} 3\right)}=\left(\frac{\mathrm{h}_{\mathrm{i}}-\mathrm{hr} 1}{\mathrm{hr} 1-\mathrm{hr} 2}\right)\left(\frac{2 \mathrm{hr} 1-\mathrm{hr} 2-1,3}{2 \mathrm{CAR}_{\mathrm{II}}}+\frac{\mathrm{h}-\mathrm{hr}_{2}}{2 \mathrm{CAR}_{\mathrm{III}}}+\frac{\mathrm{d}_{1,3}}{4}\right) \frac{\mathrm{h}_{\mathrm{i}}+\mathrm{hr} 1-2,6}{\mathrm{CAR}_{\mathrm{II}}}+\mathrm{d}_{1,3}(34) \mathrm{e} \\
& \hat{\mathrm{d}}_{\left(\mathrm{hr} 2<\mathrm{h}_{\mathrm{i}} \leq \mathrm{h}\right)}=\left(\frac{\mathrm{h}_{\mathrm{i}}-\mathrm{h}}{\mathrm{hr} 3-\mathrm{h}}\right)\left[\left(\frac{\mathrm{hr} 3-\mathrm{hrl}}{\mathrm{hr} 1-\mathrm{hr} 2}\right)\left(\frac{2 \mathrm{hrl}-\mathrm{hr} 2-1,3}{2 \mathrm{CAR}_{\mathrm{II}}}+\frac{\mathrm{h}-\mathrm{hr} 2}{2 \mathrm{CAR}_{\mathrm{III}}}+\frac{\mathrm{d}_{1,3}}{4}\right)+\frac{\mathrm{hr} 3+\mathrm{hr} 1-2,6}{\mathrm{CAR}_{\mathrm{II}}}+\mathrm{d}_{1,3}\right](35) . \\
& \text { Onde: } \quad \mathrm{CAR}_{\mathrm{I}}=\frac{2\left(1,3-\mathrm{h}_{0,0}\right)}{\left(\mathrm{d}_{1,3}-\mathrm{d}_{\mathrm{h}_{0,0}}\right)}, \quad \mathrm{CAR}_{\mathrm{II}}=\frac{2(1,3-\mathrm{hr})}{\left(\mathrm{d}_{1,3}-\mathrm{dr}\right)}, \quad \mathrm{CAR}_{\mathrm{III}}=\frac{2(\mathrm{hr}-\mathrm{h})}{\mathrm{dr}} \text {, } \\
& \mathrm{hr}=\frac{(\mathrm{h}-2)}{2}, \mathrm{hr} 1=\frac{(\mathrm{h}-1,7)}{1,7}, \mathrm{hr} 2=\frac{(\mathrm{h}-1,4)}{1,4} \mathrm{e} \mathrm{hr} 3=\frac{(\mathrm{h}-1,1)}{1,1}
\end{aligned}
$$


A avaliação do nível de acurácia das equações de perfil do tronco geradas, envolvendo as 1.297 árvores, foi feita por meio da análise da distribuição dos resíduos (equações 6 e 7) e dos seguintes critérios estatísticos:

$$
\begin{aligned}
& D P M=\left(\frac{1}{n}\right)\left\{\left[\sum_{i=1}^{n}\left[\left(y_{i}-\hat{y_{i}}\right) / y_{i}\right] 100\right]\right\}, \\
& s(y \hat{y})= \pm\left[\sqrt{\left.\sum_{i=1}^{n}\left(y_{i}-\hat{y}_{i}\right)^{2} /(n-2) / \bar{y}\right] 100} \mathrm{e}\right. \\
& P= \pm \sqrt{\left(196^{2} / \chi_{n}^{2}\right) \sum_{i=1}^{n}\left[\left(y_{i}-y_{i}\right) / y_{i}\right]^{2}} .
\end{aligned}
$$

Em que: DPM = desvio percentual médio (\%), $\mathrm{s}(\mathrm{y} y)$ = erro padrão residual (\%), $\mathrm{P}=$ exatidão obtida pelo teste de qui-quadrado $\left(\chi_{\mathrm{n}}^{2}\right)$ com nível de 0,05 (Paula Neto et al., 1983); $\hat{\mathrm{y}}_{\mathrm{i}}$ e $\mathrm{y}_{\mathrm{i}}=$ valor estimado/predito e real, respectivamente; $\mathrm{n}=$ número de pares de $\hat{\mathrm{y}_{\mathrm{i}}}$ e $\mathrm{y}_{\mathrm{i}}$; e $\overline{\mathrm{y}}=$ valor real médio.

Para facilitar a decisão quanto às equações de perfil do tronco geradas com os dados das 1.297 árvores-amostra, utilizou-se as estatísticas DPM (valor positivo), s(y y) e P resumidas em um percentual médio obtido por:

$$
p m=\frac{|D P M|+s(y \hat{y})+P}{3}
$$

As equações de perfil do tronco geradas foram, também, avaliadas em um teste de aplicação com 65 árvores-amostra cubadas pelo método de Smalian, as quais não fazem parte das 1.297 árvores-amostra empregadas para gerar equações de perfil do tronco a partir da equação de sólidos geométricos. No referido teste de aplicação, adotou-se os critérios estatísticos anteriormente apresentados e, também, um delineamento inteiramente casualizado em parcelas subdivididas, onde as parcelas foram às classes de diâmetro e altura, as subparcelas os tratamentos, isto é, os métodos avaliados e o valor real de diâmetro do tronco. Já, como repetições, foram utilizadas cinco árvores dentro de cada parcela, sendo o valor real considerado como controle. Nesta ocasião, obteve-se a porcentagem de parcelas não significativas $\left(\mathrm{PARC}^{\text {ns }}\right)$ ao nível de 0,05 pelo teste de Dunnett (Banzatto \& Kronka, 2006) e seu respectivo erro médio (Dunnett Medio Porcentual - DMP).

\section{Resultados e discussão}

As informações das 1.297 árvores-amostra cubadas foram utilizadas para vincular PD's à descrição do perfil do tronco de árvores medindo-se apenas dois diâmetros com árvores em pé. Foram testadas três possibilidades denominadas de: PDOSM2D, PDOCM2D e PDOCM2Dhv. Dentre estas, ao se usar PDOSM2D, deve-se considerar as informações apresentadas na Tabela 1, das quais, para fins de melhor entendimento, se apresentam a seguir as equações de perfil do tronco geradas na classe com diâmetro de $6,5 \mathrm{~cm}$ :

$$
\begin{aligned}
& \hat{d}_{\left(h_{0,0} \leq h_{j} \leq 1,3\right)}=\left(\frac{d_{1,3}}{4}\right)\left[\left(\frac{1,32695 d_{1,3}-h_{i}}{1,32695 d_{1,3}-1,3}\right)^{1,5069}+\left(\frac{1,3+\exp \left(0,29322 d_{1,3}\right)}{h_{i}+\exp \left(0,29322 d_{1,3}\right)}\right)^{1,50669}\right]+\left(\frac{d_{1,3}}{2}\right)\left(\frac{1,3+\exp \left(-0,13409 d_{1,3}\right)}{h_{i}+\exp \left(-0,13409 d_{1,3}\right)}\right)^{0,20268} \\
& \hat{d}_{\left(1,3<h_{i} \leq h h_{1}\right)}=\left(\frac{d_{1,3}}{2}\right)\left\{\left[\frac{(h-1,19021) / 1,19021-h_{i}}{(h-1,19021) / 1,19021-1,3}\right]^{0,48391}+\left(\frac{\exp \left(0,26933 d_{1,3}\right)}{h_{i}+\exp \left(0,26933 d_{1,3}\right)-1,3}\right)^{0,48391}\right.
\end{aligned}
$$




$$
\begin{gathered}
\hat{d}_{\left(h r 1<h_{i} \leq h r 2\right)}=d r 1\{[ \\
\hat{d}_{\left(h r 2<h_{i} \leq h\right)}=d r 2\left[\left(h-h_{i}\right) /(h-h r 2)\right]^{0,67844}
\end{gathered}
$$

Por outro lado, ao usar PDOCM2D, se deve aplicar as informações da Tabela 1 nas equações selecionadas para cada porção do tronco. Para fins de melhor entendimento, se apresentam a seguir as equações de perfil do tronco da classe com diâmetro de $6,5 \mathrm{~cm}$ :

$$
\begin{gathered}
\hat{d}_{\left(h_{0,0} \leq h_{i} \leq 1,3\right)}=\left(\frac{d_{1,3}}{4}\right)\left[\left(\frac{1,32695 d_{1,3}-h_{i}}{1,32695 d_{1,3}-1,3}\right)^{r b}+\left(\frac{1,3+\exp \left(0,29322 d_{1,3}\right)}{h_{i}+\exp \left(0,29322 d_{1,3}\right)}\right)^{r b}+\left(\frac{d_{1,3}}{2}\right)\left(\frac{1,3+e^{-0,13409 d_{1,3}}}{\left.h_{i}+e^{-0,13409 d_{1,3}}\right)^{r a}}\right.\right. \\
\hat{d}_{\left(1,3<h_{i} \leq h r 1\right)}=\left(\frac{d_{1,3}}{2}\right)\left\{\left[\frac{(h-1,19021) / 1,19021-h_{i}}{(h-1,19021) / 1,19021-1,3}\right]^{r X}+\left(\frac{\exp \left(0,26933 d_{1,3}\right)}{h_{i}+\exp \left(0,26933 d_{1,3}\right)-1,3}\right)^{r \varphi_{2}}\right\} \\
\hat{d}_{\left(h r 1<h_{i} \leq h r 2\right)}=d r 1\left\{\left[(h-1,001) / 1,001-h_{i}\right] /[(h-1,001) / 1,001-h r 1]\right\}^{r X} \\
\hat{d}_{\left(h r 2<h_{i} \leq h\right)}=d r 2\left[\left(h-h_{i}\right) /(h-h r 2)\right]^{0,678}
\end{gathered}
$$

Onde:

$$
\begin{aligned}
& r a=\left[\operatorname{Ln}\left(d_{0,3}\right)-\operatorname{Ln}\left(d_{1,3}\right)\right] /\left[\operatorname{Ln}\left(1,3+\exp \left(-0,13409 d_{1,3}\right)\right)-\operatorname{Ln}\left(0,3+\exp \left(-0,13409 d_{1,3}\right)\right)\right], \\
& r b=\left[\operatorname{Ln}\left(d_{0,3}\right)-\operatorname{Ln}\left(d_{1,3}\right)\right] /\left[\operatorname{Ln}\left(1,32695 d_{1,3}-0,3\right)-\operatorname{Ln}\left(1,32695 d_{1,3}-1,3\right)\right] \mathrm{e} \\
& r_{b_{j}}^{\prime}=\left[\operatorname{Ln}\left(d_{0,3_{j}}\right)-\operatorname{Ln}\left(d_{1,3_{j}}\right)\right] /\left\{\operatorname{Ln}\left[1,3+\exp \left(0,29322 d_{1,3_{j}}\right)\right]-\operatorname{Ln}\left[0,3+\exp \left(0,29322 d_{1,3_{j}}\right)\right]\right\} .
\end{aligned}
$$

Na equação 41, usada na PMT, e $\mathrm{r} \phi_{2_{\mathrm{j}}}$, são calculados para a classe com diâmetro de 6,5 cm, empregando-se:

$$
\begin{aligned}
& \mathrm{rX}=\left[\operatorname{Ln}(\mathrm{dra})-\operatorname{Ln}\left(\mathrm{d}_{1,3}\right)\right] /\{\operatorname{Ln}[(\mathrm{h}-1,19021) / 1,19021-\mathrm{hra}]-\operatorname{Ln}[(\mathrm{h}-1,19021) / 1,19021-1,3]\}, \\
& h r a=(h-2,5) / 2,5 \mathrm{e} \\
& \mathrm{r} \phi_{2}=\left[\operatorname{Ln}(\mathrm{dra})-\operatorname{Ln}\left(\mathrm{d}_{1,3}\right)\right] /\left\{\operatorname{Ln}\left[\exp \left(0,26933 \mathrm{~d}_{1,3}\right)\right]-\operatorname{Ln}\left[\mathrm{hra}+\exp \left(0,26933 \mathrm{~d}_{1,3}\right)-1,3\right]\right\} .
\end{aligned}
$$

Na equação $42, d r_{1_{j}}$ é obtido por meio da equação empregada na PMT (equação 41) substituindo-se $h_{i}$ por hr1 e rX é calculado na PST para cada classe com diâmetro de $6,5 \mathrm{~cm}$, empregando-se:

$$
\begin{aligned}
& \mathrm{rX}=[\operatorname{Ln}(\mathrm{drb})-\operatorname{Ln}(\mathrm{drl})] / \operatorname{Ln}[(\mathrm{h}-1,00983) / 1,00983-\mathrm{hrb}]-\operatorname{Ln}[(\mathrm{h}-1,00983) / 1,00983-\mathrm{hrl}] \\
& \mathrm{hrl}=\frac{\mathrm{h}_{\mathrm{j}}-1,4}{1,4} \text { e } \mathrm{hrb}=\left(\mathrm{h}_{\mathrm{j}}-1,25\right) / 1,25 .
\end{aligned}
$$


Tabela 1. Tabela de parâmetros de equações de perfil do tronco referente aos protótipos dendrométricos otimizados obtidos para diferentes segmentos do tronco e classes de diâmetro.

\begin{tabular}{|c|c|c|c|c|}
\hline \multirow{3}{*}{ Classe $d_{1,3}$} & \multicolumn{4}{|c|}{ Porção do tronco } \\
\hline & $\begin{array}{c}\text { PBT } \\
(0,0 \text { a } 1,3)\end{array}$ & $\begin{array}{c}\text { PMT } \\
\left(1,3 a \mathbf{h r}_{1}\right)\end{array}$ & $\begin{array}{c}\text { PST } \\
\left(h_{1} \mathbf{a ~ h r}_{2}\right)\end{array}$ & \multirow{2}{*}{$\begin{array}{c}\text { PAT } \\
\left(\mathbf{h r}_{\mathbf{2}} \mathbf{a} \mathbf{h}\right) \\
\mathrm{ra}=0,67844\end{array}$} \\
\hline & $\phi_{1} \mathrm{~cm}=1,32695$ & $\mathrm{Xm}=1,19021$ & $\mathrm{Xa}=1,00983$ & \\
\hline \multirow{4}{*}{$\begin{array}{c}6,5 \\
\text { (média } \\
\text { entre } \\
12 \text { e } 14 \text { ) }\end{array}$} & $\phi_{2} \mathrm{~cm}=0,29322$ & $\phi_{2} \mathrm{~cm}=0,26933$ & $\mathrm{rc}=0,42070$ & \\
\hline & $\mathrm{rma}=1,50569$ & $\mathrm{rma}=0,48391$ & Equação (21) & Equação (23) \\
\hline & $\phi_{2} \mathrm{c}=-0,13409$ & Equação (19) & & \\
\hline & $\mathrm{rc}=0,20268$ & & & \\
\hline \multirow{5}{*}{$\begin{array}{c}9,5 \\
\text { (média } \\
\text { entre } \\
12 \text { e } 14 \text { ) }\end{array}$} & $\phi_{1} \mathrm{~cm}=1,00699$ & $\mathrm{Xm}=1,28967$ & $\mathrm{Xm}=1,00000$ & $\mathrm{ra}=0,76113$ \\
\hline & $\phi_{2} \mathrm{~cm}=0,21531$ & $\phi_{2} \mathrm{~cm}=0,22706$ & $\phi_{2} \mathrm{~cm}=0,09516$ & \\
\hline & $\mathrm{rma}=1,44644$ & $\mathrm{rma}=0,50562$ & $\mathrm{rma}=0,51251$ & Equação (23) \\
\hline & $\phi_{2} \mathrm{c}=-0,07540$ & Equação (19) & Equação (22) & \\
\hline & $\mathrm{rc}=0,19850$ & & & \\
\hline \multirow{5}{*}{$\begin{array}{c}12,5 \\
\text { (média } \\
\text { entre } \\
12 \text { e } 14 \text { ) }\end{array}$} & $\phi_{1} \mathrm{~cm}=0,81752$ & $X=0,71645$ & $\mathrm{Xa}=0,80466$ & $\mathrm{ra}=0,79828$ \\
\hline & $\phi_{2} \mathrm{~cm}=0,17206$ & $\mathrm{rca}=1,05345$ & $\mathrm{rc}=1,13172$ & \\
\hline & $\mathrm{rma}=1,50214$ & Equação (17) & Equação (48) & Equação (23) \\
\hline & $\phi_{2} \mathrm{c}=-0,05154$ & & & \\
\hline & $\mathrm{rc}=0,19863$ & & & \\
\hline \multirow{5}{*}{$\begin{array}{c}15,5 \\
\text { (média } \\
\text { entre } \\
12 \text { e } 14 \text { ) }\end{array}$} & $\phi_{1} \mathrm{~cm}=0,74560$ & $\mathrm{Xm}=1,15252$ & $\mathrm{Xa}=0,87991$ & $\mathrm{ra}=0,85661$ \\
\hline & $\phi_{2} \mathrm{~cm}=0,14730$ & $\phi_{2} \mathrm{~cm}=0,59834$ & $\mathrm{rc}=1,12469$ & \\
\hline & $\mathrm{rma}=1,51796$ & $\mathrm{rma}=0,14145$ & Equação (21) & Equação (23) \\
\hline & $\phi_{2} \mathrm{c}=-0,02461$ & Equação (19) & & \\
\hline & $\mathrm{rc}=0,19950$ & & & \\
\hline \multirow{5}{*}{$\begin{array}{c}18,5 \\
\text { (média } \\
\text { entre } \\
12 \text { e } 14 \text { ) }\end{array}$} & $\phi_{1} \mathrm{~cm}=0,66501$ & $\mathrm{Xa}=0,75766$ & $X=0,89922$ & $\mathrm{ra}=0,89757$ \\
\hline & $\phi_{2} \mathrm{~cm}=0,12853$ & $\mathrm{rc}=1,05641$ & rca $=0,90297$ & \\
\hline & $\mathrm{rma}=1,46908$ & Equação (18) & Equação (20) & Equação (23) \\
\hline & $\phi_{2} \mathrm{c}=-0,01397$ & & & \\
\hline & $\mathrm{rc}=0,19886$ & & & \\
\hline \multirow{5}{*}{$\begin{array}{c}21,5 \\
\text { (Média } \\
\text { entre } \\
12 \text { e } 14 \text { ) }\end{array}$} & $\phi_{1} \mathrm{~cm}=0,57668$ & $\mathrm{Xm}=1,19575$ & $X=0,90000$ & $\mathrm{ra}=0,91779$ \\
\hline & $\phi_{2} \mathrm{~cm}=0,11087$ & $\phi_{2} \mathrm{~cm}=0,59801$ & $\mathrm{rca}=0,90156$ & \\
\hline & $\mathrm{rma}=1,49398$ & $\mathrm{rma}=0,11482$ & & Equação (23) \\
\hline & $\phi_{2} \mathrm{c}=-0,01026$ & Equação (19); & & \\
\hline & $\mathrm{rc}=0,19860$ & também em 24,5 e 27,5 & & \\
\hline \multirow{3}{*}{$\begin{array}{c}24,5 \\
\text { (equação } \\
13)\end{array}$} & $\phi_{1} \mathrm{c}=0,11274$ & $\mathrm{Xm}=1,05287$ & $X=0,79631$ & $\mathrm{ra}=0,98822$ \\
\hline & $\phi_{2} \mathrm{c}=0,00480$ & $\phi_{2} \mathrm{~cm}=0,68705$ & rca $=1,45847$ & Equação (23) \\
\hline & $\mathrm{rmb}=0,20048$ & $\mathrm{rma}=0,10466$ & Equação (20) & \\
\hline \multirow{3}{*}{$\begin{array}{c}27,5 \\
\text { (equação } \\
12)\end{array}$} & $\phi_{2} \mathrm{c}=0,03065$ & $\mathrm{Xm}=1,13703$ & $X=0,70000$ & $\mathrm{ra}=0,80276$ \\
\hline & $\phi_{2} \mathrm{c}=0,32810$ & $\phi_{2} \mathrm{~cm}=0,66418$ & $\mathrm{rca}=1,84796$ & Equação (23) \\
\hline & & $\mathrm{rma}=0,09517$ & Equação (20) & \\
\hline
\end{tabular}


Por fim, na equação 43, dr2 é obtido por meio da equação empregada na PST (equação 42) substituindose $h_{\mathrm{i}}$ por $\mathrm{hr} 2=(\mathrm{h}-1,1) / 1,1$.

As equações de perfil do tronco das demais classes de diâmetro são obtidas da mesma forma a partir da
Tabela 1, vinculando o PDO próprio de cada árvore à medição dos diâmetros situados em $0,3 \mathrm{~m}, 1,3 \mathrm{~m}$, hra $=(\mathrm{h}-2) / 2$ e hrb $=(\mathrm{h}-1,25) / 1,25$. Ressalta-se, para uso destas equações, consultar Andrade (2006) onde se apresentam os detalhes de todas as equações de perfil do tronco geradas neste estudo.

Na possibilidade de uso do PDOCM2Dhv, se deve usar as seguintes equações de perfil do tronco:

$$
\begin{aligned}
& \hat{\mathrm{d}}_{\left(0,0 \leq \mathrm{h}_{\mathrm{i}} \leq 1,3\right)}=\mathrm{d}_{1,3}\left[\left(\mathrm{hv}+0,28663-\mathrm{h}_{\mathrm{i}}\right) /(\mathrm{hv}+0,28663-1,3)\right]^{(3 / 2)}(44), \\
& \hat{\mathrm{d}}_{\left(1,3 \leq \mathrm{h}_{\mathrm{i}} \leq \mathrm{hr} 1\right)}=\mathrm{d}_{1,3}\left[\left(\mathrm{hv}+1,19731-\mathrm{h}_{\mathrm{i}}\right) /(\mathrm{hv}+1,19731-1,3)\right]^{(1,2)}(45), \\
& \hat{\mathrm{d}}_{\left(\mathrm{hr} 1 \leq \mathrm{h}_{\mathrm{i}} \leq \mathrm{hr} 2\right)}=\mathrm{d}_{1,3}\left[\left(\mathrm{hv}-0,19251-\mathrm{h}_{\mathrm{i}}\right) /(\mathrm{hv}-0,19251-1,3)\right]^{(0,8)}(46) \mathrm{e} \\
& \hat{\mathrm{d}}_{\left(\mathrm{hr} 2 \leq \mathrm{h}_{\mathrm{i}} \leq \mathrm{h}\right)}=\mathrm{dr} 2\left[\left(\mathrm{~h}-\mathrm{h}_{\mathrm{i}}\right) /(\mathrm{h}-\mathrm{hr} 2)\right](47) .
\end{aligned}
$$

Onde:

$$
\begin{aligned}
& \text { hv }=\left\lfloor 0,3-1,3\left(\mathrm{~d}_{0,3} / \mathrm{d}_{1,3}\right)^{(0,67)}\right\rfloor /\left[1-\left(\mathrm{d}_{0,3} / \mathrm{d}_{1,3}\right)^{(0,67)}\right\rfloor \text { para a equação } 44, \\
& \mathrm{hv}=\left\lfloor\mathrm{hrb}-1,3\left(\mathrm{drb} / \mathrm{d}_{1,3}\right)^{(0,83)}\right\rfloor /\left[1-\left(\mathrm{drb} / \mathrm{d}_{1,3}\right)^{(0,83)}\right\rfloor \text { para a equação } 45, \text { e } \\
& \text { hv }=\left\lfloor\mathrm{hrb}-1,3\left(\mathrm{drb} / \mathrm{d}_{1,3}\right)^{1,25}\right\rfloor /\left[1-\left(\mathrm{drb} / \mathrm{d}_{1,3}\right)^{1,25}\right\rfloor \text { para a equação } 46 .
\end{aligned}
$$

Nas equações 44, 45 e 46, nota-se que $\mathrm{hv}_{\mathrm{j}}$ é obtido para PBT, PMT e PST de cada i-ésima árvore, independentemente da classe de diâmetro considerada. Para isto, utilizam-se os diâmetros situados à $0,3 \mathrm{~m}, 1,3 \mathrm{~m}$ e em hrb=(h-1,25)/1,25 $\mathrm{m}$ do terreno, respectivamente. Já, para a equação 47, dr2 é obtido empregando-se a equação 46 substituindo $\mathrm{h}_{\mathrm{i}}$ por hr2.

As três possibilidades de se descrever o perfil do tronco de árvores: PDOSM2D, PDOCM2D e PDOCM2Dhv, juntamente com AR, foram aplicadas em 1.297 árvores, gerando-se os resultados apresentados na Tabela 2. Nota-se na PBT que o método PDOSM2D apresentou, expressivamente, os piores resultados com um $\mathrm{pm}=6,1 \%$ contra AR, PDOCM2D e PDOCM2Dhv com pm em torno de 3,6\%. Assim, nota-se que é adequado usar AR, PDOCM2D e PDOCM2Dhv na descrição do perfil do tronco na PBT.
Por outro lado, na PMT observa-se AR e PDOCM2Dhv com pm menor que $4 \%$ contra PDOSM2D e PDOCM2D com pm superior à 5,5\%. Assim, tanto AR como PDOCM2Dhv são os mais adequados para descrever o perfil do tronco na PMT. Já, na PST, a melhor opção, de forma expressiva, é o PDOCM2Dhv, com pm $=6,9 \%$, seguido de AR com pm $=12,4 \%$, sendo PDOCM2D e PDOSM2D as piores opções. Por fim, na PAT, observa-se que a melhor opção para descrever o perfil do tronco é o AR seguido de PDOSM2D.

Diante disso, o PDOCM2D só é adequado para uso na PBT e o PDOSM2D apenas na PST. Já, o PDOCM2Dhv é adequado para uso na PBT, PMT e PST, sendo AR uma opção de uso para qualquer que seja a porção do tronco. Assim, além de AR, organizou-se três outros métodos para descrever o perfil do tronco inteiro de árvores de eucalipto, os quais resultaram nos métodos apresentados na Tabela 3. 
Tabela 2. Estatísticas em percentagem, que expressam a confiabilidade de se usar um PDO para descrever o perfil do tronco empregando-se dados de 1297 árvores-amostra cubadas por Smalian.

\begin{tabular}{|c|c|c|c|c|c|}
\hline Porção tronco & Método & DPM & $s(y \hat{y})$ & $\mathbf{P}$ & pm \\
\hline \multirow{4}{*}{ PBT - Porção basal do tronco } & $\mathrm{AR}$ & 0,498 & 3,8 & 7,0 & 3,75 \\
\hline & SPDOSM2D & 0,352 & 5,3 & 12,7 & 6,10 \\
\hline & SPDOCM2D & 0,611 & 3,6 & 6,6 & 3,60 \\
\hline & SPDOCM2Dhv & $-0,00011$ & 3,8 & 6,9 & 3,55 \\
\hline \multirow{4}{*}{ PMT - Porção mediana do tronco } & $\mathrm{AR}$ & 0,261 & 3,5 & 8,1 & 3,95 \\
\hline & SPDOSM2D & 0,065 & 5,3 & 11,2 & 5,53 \\
\hline & SPDOCM2D & 0,024 & 5,6 & 18,5 & 8,04 \\
\hline & SPDOCM2Dhv & 0,0000146 & 4,0 & 8,0 & 3,98 \\
\hline \multirow{4}{*}{ PST - Porção superior do tronco } & $\mathrm{AR}$ & $-2,106$ & 11,1 & 23,9 & 12,38 \\
\hline & SPDOSM2D & $-0,453$ & 12,5 & 26,9 & 14,89 \\
\hline & SPDOCM2D & 3,078 & 16,5 & 43,0 & 20,88 \\
\hline & SPDOCM2Dhv & 0,0002349 & 6,4 & 14,1 & 6,85 \\
\hline \multirow{4}{*}{ PAT - Porção apical do tronco } & $\mathrm{AR}$ & $-2,909$ & 22,0 & 49,6 & 24,84 \\
\hline & SPDOSM2D & 3,466 & 24,2 & 52,2 & 26,62 \\
\hline & SPDOCM2D & 14,487 & 26,6 & 76,9 & 39,32 \\
\hline & SPDOCM2Dhv & $-1,526$ & 23,9 & 63,6 & 29,66 \\
\hline
\end{tabular}

Tabela 3. Métodos selecionados em cada porção do tronco a partir dos resultados apresentados na Tabela 2.

\begin{tabular}{ccccc}
\hline Método & PBT $\left(\mathbf{h}_{\mathbf{i}} \leq \mathbf{1}, \mathbf{3} \mathbf{~ m}\right)$ & PMT $\left(\mathbf{h}_{\mathbf{i}} \leq \mathbf{h} \mathbf{r} \mathbf{~} \mathbf{m}\right)$ & PST $\left(\mathbf{h}_{\mathbf{i}} \leq \mathbf{h r} \mathbf{2} \mathbf{~ m}\right)$ & PAT $\left(\mathbf{h}_{\mathbf{i}} \leq \mathbf{h} \mathbf{~ m}\right)$ \\
\hline I & AR & AR & AR & AR \\
II & SPDOCMDhv & AR & SPDOCMDhv & AR \\
III & SPDOCMDhv & SPDOCMDhv & SPDOCMDhv & AR \\
IV & SPDOCM2D & AR & SPDOCMDhv & AR \\
\hline
\end{tabular}

Na Tabela 4 nota-se que os métodos I e III são os piores para perfil do tronco em comparação com os métodos II e IV, onde se observam $76,9 \%$ das parcelas sendo não significativas pelo teste Dunnett, com $\mathrm{DMP}=0,41 \%$ e DMP $=-0,14 \%$ contra $92,3 \%$ de parcelas não significativas dos métodos II e IV com DMP de $0,12 \%$ e $0,13 \%$, respectivamente.

Houve, também, expressiva superioridade de $\mathrm{s}(\mathrm{y} y)$ e $\mathrm{P}$ com valores inferiores à $\pm 3,8 \%$ e em torno de $\pm 10 \%$, respectivamente. Dentre os métodos II e IV, notam-se resultados bastante semelhantes na descrição do perfil do tronco, sugerindo a preferência pelo método II por empregar PDOCM2Dhv na PBT, o qual é de mais fácil desenvolvimento que o PDOCM2D (Tabela 3).
Tabela 4. Resultados dos critérios estatísticos adotados para avaliar quatro métodos de empregar protótipos dendrométricos na descrição do perfil do tronco e quantificação do volume total em 65 árvores-amostras.

\begin{tabular}{|c|c|c|c|c|}
\hline Estatística & MI & MII & MIII & MIV \\
\hline \multicolumn{5}{|c|}{ Perfil do tronco $\left(d_{i}\right), n=1.339$ posições do tronco: } \\
\hline DMP & 0,41 & 0,12 & $-0,14$ & 0,13 \\
\hline $\mathrm{PARC}^{\mathrm{ns}}$ & 76,90 & 92,30 & 76,90 & 92,30 \\
\hline s(yy) & 4,25 & 3,85 & 4,89 & 3,71 \\
\hline $\mathrm{P}(\%)$ & 12,83 & 10,44 & 18,02 & 10,38 \\
\hline \multicolumn{5}{|c|}{ Volume total (vt), $n=65$ árvores: } \\
\hline DMP & 1,00 & 0,62 & 0,25 & 0,63 \\
\hline PARC $^{\text {ns }}$ & 69,23 & 76,92 & 76,92 & 76,92 \\
\hline$s(\hat{y})$ & 3,76 & 2,84 & 4,71 & 2,84 \\
\hline $\mathrm{P}(\%)$ & 6,59 & 4,85 & 6,64 & 4,91 \\
\hline
\end{tabular}


$\mathrm{Na}$ análise para volume total (Tabela 4), se observa o mesmo comportamento quando da análise do perfil do tronco: superioridade dos métodos II e IV frente aos métodos I e III, principalmente, quanto à $\mathrm{s}(\mathrm{y} \mathrm{y})$ e $\mathrm{P}$, com valores inferiores à $\pm 2,8 \% \mathrm{e} \pm 4,9 \%$ contra valores superiores a $\pm 3,8 \%$ e $\pm 6,5 \%$, respectivamente. Dentre os métodos II e IV, deve-se escolher o método II por ser de mais fácil desenvolvimento, como referido na análise do perfil do tronco.

De maneira geral, se observa que os resultados da Tabela 4 são similares aos obtidos por Andrade \& Leite (2001), Andrade et al. (2006) e Carvalho et al. (2010), onde $\mathrm{s}(\mathrm{y} y)$ oscilou entre 3,8 e 12,3\% bem superior aos valores obtidos em trabalhos com o uso da cubagem rigorosa tradicional, onde $\mathrm{s}(\mathrm{y} y)$ oscilou entre $5,1 \mathrm{e}$ $20,5 \%$, cujos melhores modelos apresentaram s(y y) em torno de $6,0 \%$, enquanto neste trabalho, se obteve s(y y) em torno de 3,7\%, cabendo citar: Müller et al. (2005), Pires \& Calegário (2007), Souza et al. (2008), Silva et al. (2011), Lanssanova et al. (2013), dentre outros. Nestes trabalhos, se observa que a cubagem rigorosa foi feita após o abate das árvores-amostra tomando-se diâmetros ao longo do tronco em várias posições. Já com o método da altura relativa poucos diâmetros são medidos, o que viabiliza não abater árvores-amostra localizadas dentro das parcelas de inventário. Assim, se observa que o método da altura relativa é bastante promissor para conduzir inventários florestais com medições de parcelas e de cubagem em simultâneo.

$\mathrm{Na}$ análise da distribuição dos resíduos obteve-se a mesma conclusão da Tabela 4. Como a decisão foi pelo método II, então, se apresenta nas Figuras 3 e 4, a distribuição de resíduos deste em comparação com o método I, que é a forma atual de desenvolvimento do método da altura relativa. Pode-se ver que o método II tem melhor distribuição de resíduos, ficando a dispersão dos pontos em $\pm 1 \mathrm{~cm}$, principalmente, para diâmetro predito menor que $20 \mathrm{~cm}$ aproximadamente (Figuras $3 \mathrm{a} \mathrm{e} 3 \mathrm{~b}$ ).
( a )

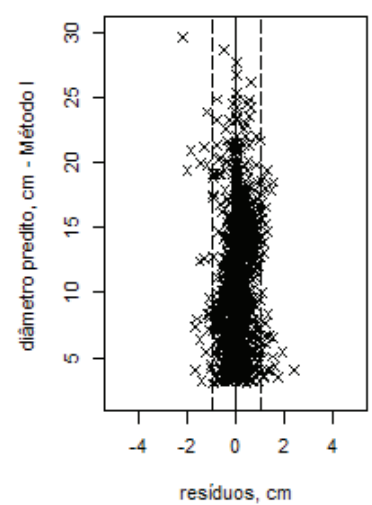

(b)

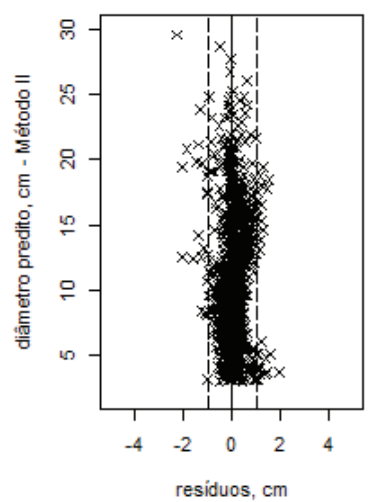

(c)

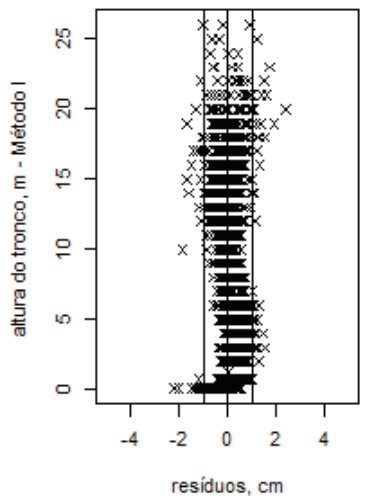

(d)

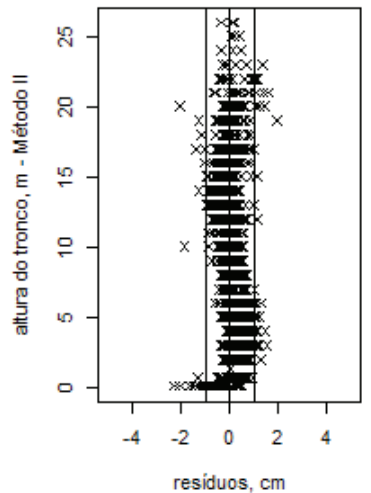

(e)

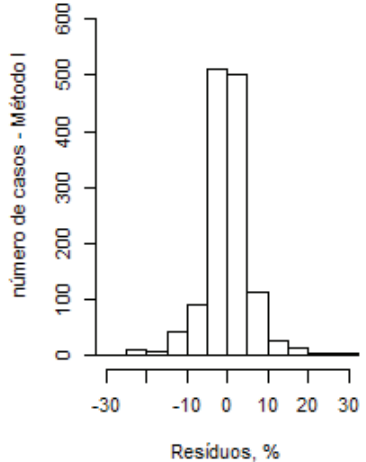

(f)

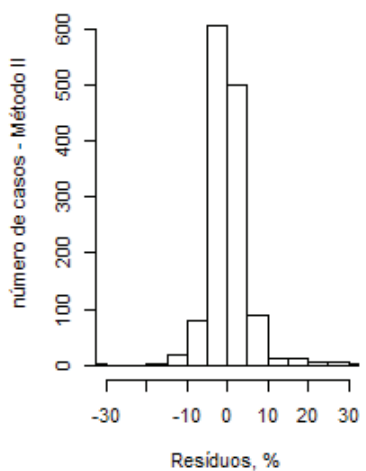

Figura 3. Distribuição de resíduos para perfil do tronco empregando-se os métodos I e II. 
( a )

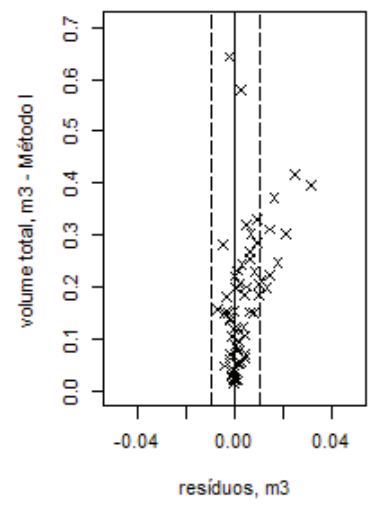

(b)

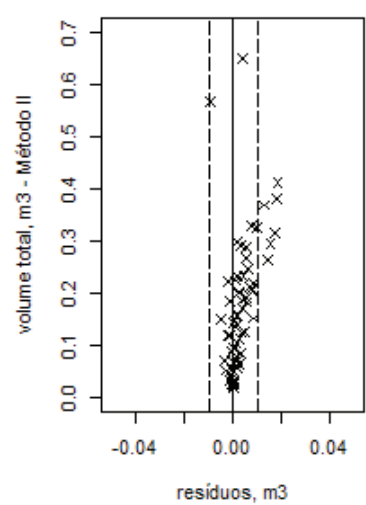

(c)

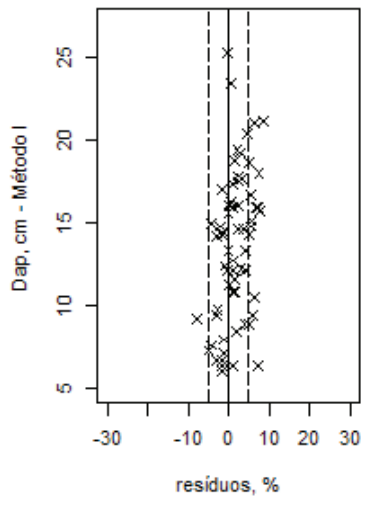

(d)

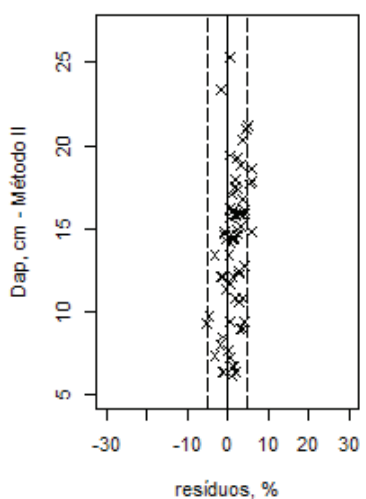

(e)

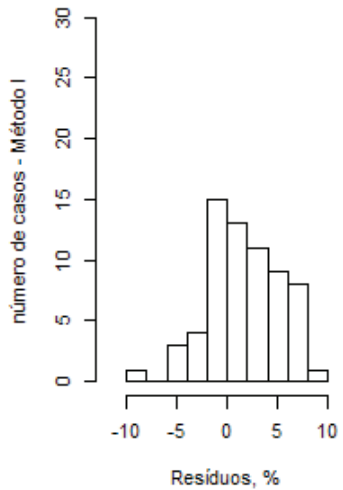

(f)

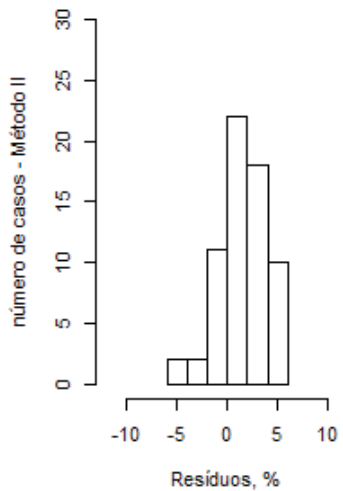

Figura 4. Distribuição de resíduos para volume total empregando-se os métodos I e II.

Nas Figuras 3c e 3d, nota-se que o método II é superior ao método I, de forma bastante expressiva para alturas acima de $15 \mathrm{~m}$. De acordo com isto, está o histograma dos resíduos porcentuais (Figuras $3 \mathrm{e}$ e 3f), onde se nota que o método II é bastante superior ao método I, tendo a maioria dos resíduos oscilando em $\pm 5 \%$, cerca de 1.100 casos contra em torno de 1.000 casos do método I, representando cerca de $82,2 \%$ e $74,7 \%$, respectivamente.

Para volume total, nota-se uma dispersão tendendo à superestimação com o aumento dos valores preditos a partir de $0,20 \mathrm{~m}^{3}$ para o método I e de $0,25 \mathrm{~m}^{3}$ para o método II (Figuras 4a e 4b). Pode-se ver que a superestimação oscila entre 16 e $22 \mathrm{~cm}$ de diâmetro para os métodos I e II (Figuras 4c e 4d). Abaixo destes intervalos, a dispersão se apresenta de forma bem distribuída e equilibrada, sendo bem melhor para o método II. Nota-se que a dispersão dos resíduos se distribuem em no máximo $\pm 5 \%$ para o método II e em $\pm 10 \%$ para o método I (Figuras 4e e 4f). Para resíduos que oscilam em $\pm 2 \%$, são 28 casos para o método I contra em torno de 32 casos do método II, representando cerca de $43,1 \%$ e $49,2 \%$, respectivamente.

Diante das análises feitas com base na Tabela $4 \mathrm{e}$ Figuras 3 e 4, pode-se ver que o método II é melhor que o método I e, portanto, pode-se considerá-lo como sendo uma nova versão de desenvolvimento do método da altura relativa. Observa-se no método II que as equações de perfil do tronco são geradas a partir da medição de dois diâmetros em alturas superiores a 1,3 $\mathrm{m}$ do terreno, sendo um diâmetro medido em $\mathrm{hra}=(\mathrm{h}-2) / 2$ e outro em $\mathrm{hrb}=(\mathrm{h}-1,25) / 1,25$.

Para um melhor entendimento do método II, apresenta-se a seguir a sequência que deve ser adotada para simular a cubagem rigorosa do tronco inteiro de árvores em pé, preferencialmente, localizadas dentro das parcelas de inventário. A sequência tem os seguintes passos: 
1) Medir o diâmetro à $0,3 \mathrm{~m}$ do terreno para gerar a seguinte equação de perfil do tronco:

$$
\hat{d}_{\left(0,0 \leq h_{i} \leq 1,3\right)}=d_{1,3}\left[\left(h v+0,28663-h_{i}\right) /(h v+0,28663-1,3)\right]^{(3 / 2)}
$$

Onde:

$$
h v=\left[0,3-1,3\left(d_{0,3} / d_{1,3}\right)^{0,67}\right] /\left[1-\left(d_{0,3} / d_{1,3}\right)^{0,67}\right]
$$

2) Medir o diâmetro em $h r a=(h-2) / 2 m$ do terreno para gerar a seguinte equação de perfil do tronco:

$$
\hat{d}_{\left(1,3<h_{i} \leq h r 1\right)}=\left(2 \cdot h_{i}-2,6\right) / C A R_{I I}+d_{1,3}
$$

Onde:

$$
C A R_{I I}=2(1,3-h r a) /\left(d_{1,3}-d r a\right) \quad \text { e } \quad h r 1=(h-1,4) / 1,4
$$

3) Medir o diâmetro em $h r b=\frac{(h-1,25)}{1,25)}$ m do terreno para gerar a seguinte equação de perfil do tronco:

$$
\hat{d}_{\left(h r 1 \leq h_{i} \leq h r 2\right)}=d_{1,3}\left[\left(h v-0,19251-h_{i}\right) /(h v-0,19251-1,3)\right]^{0,80}
$$

Onde:

$$
h r 2=(h-1,1) / 1,1 \text { e } h v=\left[h r b-1,3\left(d r b / d_{1,3}\right)^{1,25}\right] /\left[1-\left(d r b / d_{1,3}\right)^{1,25}\right]
$$

4) Usar a seguinte equação de perfil do tronco na PAT:

Onde:

$$
\hat{d}_{\left(h r 2<h_{i} \leq h\right)}=\left(\frac{h_{i}-h}{h r 2-h}\right)\left[\left(\frac{h r 2-h r c}{h r c-h r 1}\right)\left(\frac{2 h r c-h r 1-1,3}{2 C A R_{I I}}+\frac{h-h r 1}{2 C A R_{I I I}}+\frac{d_{1,3}}{4}\right)+\frac{h r 2+h r c-2,6}{C A R_{I I}}+d_{1,3}\right]
$$

$C A R_{I I}=2(1,3-h r a) /\left(d_{1,3}-d r a\right), C A R_{I I I}=2(h r a-h) / d r a \quad$ e $\quad h r c=(h-1,7) / 1,7$

As análises feitas permitem inferir que um PDO pode ser determinado medindo-se somente os diâmetros referentes às posições que o delimitam no tronco das árvores. Não é necessário abater árvores para cubar a fim de se ter os dados necessários à estimativa de equações de perfil do tronco, conforme se faz usualmente, obtendo-se em árvores-amostra abatidas e em separado das parcelas. No emprego dos métodos I e II, visualiza-se um cenário onde se podem medir diâmetros com árvores em pé e localizadas dentro das parcelas de inventário, em simultâneo com as demais medições.
A importância dos métodos I e II é ilustrada na Figura 5 onde se observa que o método usual de cubagem rigorosa (Figura 5a), em média, exige a medição de 20,6 diâmetros ao longo do tronco de árvores abatidas. No método I (Figura 5b), seriam necessários medir três diâmetros com as árvores em pé, o que reduz o número de diâmetros medidos ao longo do tronco por ocasião da cubagem em cerca de $85 \%$. Já no método II (Figura $5 \mathrm{c}$ ), este valor cai para cerca de $80 \%$, porque se exige a medição de quatro diâmetros no tronco de árvores em pé. 

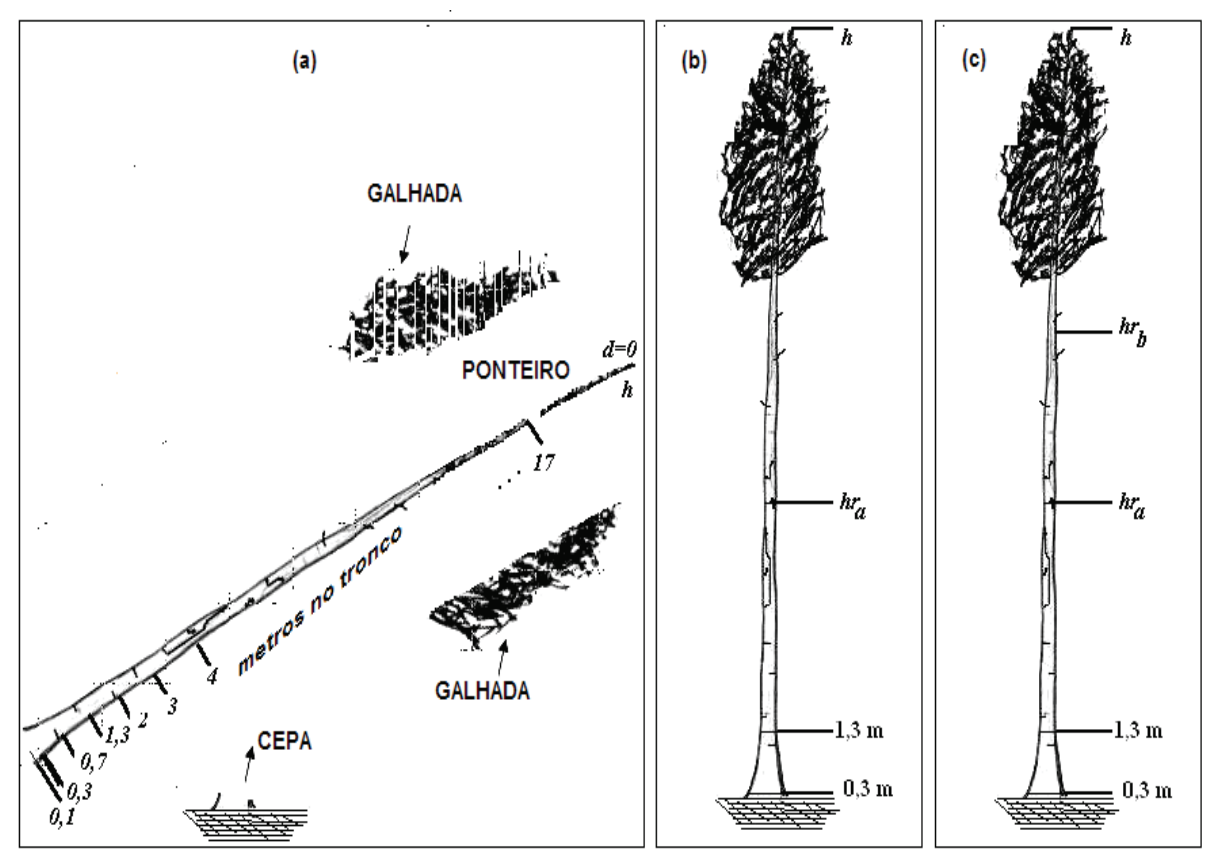

Figura 5. Esquema de uma cubagem empregando o método usual(a), o método I (b) e o método II (c).

\section{Conclusões}

A equação de um protótipo dendrométrico otimizado representa uma equação de perfil do tronco para quantificar multiprodutos da madeira de árvores em pé.

Observou-se que:

- Deve-se medir diâmetros do tronco em 0,3, 1,3, (h-2)/2 e em (h-1,25)/1,25 além de h;

- Deve-se usar, preferencialmente, PDOCM2Dhv na porção basal do tronco ao invés do PDOCM2D;

- Deve-se usar, preferencialmente, AR na porção mediana do tronco;

- Deve-se usar, preferencialmente, PDOCM2Dhv na porção superior do tronco;

- Deve-se usar, preferencialmente, AR na porção apical do tronco; e

- Deve-se desenvolver o PDOCM2Dhv medindo-se drb em (h-1,25)/1,25 $\mathrm{m}$ do terreno.

O método I reduz em $85 \%$ e o II em $80 \%$ a quantidade de diâmetros à medir no tronco de árvores em pé para gerar equações de perfil do tronco;

$\mathrm{O}$ método II tem maior potencial de substituir os métodos usuais que o método I para quantificar multiprodutos da madeira. Por isso, decidiu-se denominá-lo: método da altura relativa com dois diâmetros (hr2D).

\section{Agradecimentos}

Á CAPES - Coordenação de Aperfeiçoamento de Pessoal de Nível Superior, pela bolsa de Doutorado concedida ao primeiro autor, viabilizando seu doutoramento na Universidade Federal de Lavras (UFLA) quando se produziu o conteúdo apresentado neste estudo.

\section{Referências}

ANDRADE, V. C. L.; CALEGÁRIO, N.; SOUZA, A. L.; REZENDE, J. L. P.; TRUGILHO, P. F.; ROSADO, S. C. S.; SILVA, R. M. Uso de sólidos geométricos para gerar equações de forma do tronco de árvores em pé. Scientia Forestalis, Piracicaba, v. 37, n. 83, p. 299$311,2009$.

ANDRADE, V. C. L.; CALEGARIO, A. N.; SCOLFORO, J. R. S. Analise de algumas alternativas para obter o coeficiente angular da reta no método da altura relativa. Ciência Florestal, Santa Maria, RS, v. 16, n. 3, p. 303-317, 2006.

ANDRADE, V. C. L.; LEITE, H. G. Uso da geometria analítica para descrever o taper quantificar o volume de árvores individuais. Revista Árvore, Viçosa, MG, v. 25, n. 4, p. 481-486, 2001.

ANDRADE, V. C. L. Cubagem de árvores em pé pelo método da altura relativa. 2006. 152 f. Tese (Doutorado em Engenharia Florestal) - Universidade Federal de Lavras, Lavras.

BANZATTO, D. A.; KRONKA, S. N. Experimentação agrícola. 4. ed. Jaboticabal: Funep, 2006. 237 p. 
CARVALHO, S. P. C.; MENDONÇA, A. R.; LIMA, M. P.; CALEGARIO, N. Different strategies to estimate the commercial volume of anadenanthera colubrine (vell.) brenan. Cerne, Lavras, v. 16, n. 3, p. 399-406, 2010.

CHICHORRO, J. F. Análise estrutural econômica de multiprodutos da madeira em florestas naturais. 2000. $241 \mathrm{f}$. Tese (Doutorado em Ciência Florestal) - Universidade Federal de Viçosa, Viçosa, MG.

COUTO, H. T. Z.; VETORAZZO, S. C. Seleção de equações de volume e peso seco comercial para Pinus taeda. Cerne, Lavras, v. 5, n. 1, p. 69-80, 1999.

GOMES, A. M. A. Medição dos arvoredos. Lisboa: Sa da Costa, 1957, 413 p. (A Terra e o Homem, 30).

LANSSANOVA, L. R.; UBIALLI, J. A.; ARCE, J. E.; PELISSARI, A. L.; FAVALESSA, C. M. C.; DRESCHER, R. Avaliação de funções de afilamento para a estimativa de diâmetro de espécies florestais comerciais do bioma amazônico mato-grossense. Floresta, Curitiba, v. 43, n. 2, p. 215-224, 2013.

LEITE, H. G.; ANDRADE, V. C. L. Um método para condução de inventários florestais sem o uso de equações volumétricas. Revista Árvore, Viçosa, MG, v. 26, n. 3, p. 321-328, 2002.
MÜLLER, I.; FINGER, C. A. G.; SCHNEIDER, P. R. Forma de tronco e sortimentos de madeira de eucalyptus grandis hill ex maiden, na região sudeste do estado do Rio Grande do Sul. Ciência Florestal, Santa Maria, RS, v. 15, n. 3, p. 293-305, 2005.

PAULA NETO, F.; SOUZA, A. L. de; QUINTAES, P. C. G.; SOARES, V. P. Análise de equações volumétricas para Eucalyptus spp., segundo o método de regeneração na região de José de Melo - MG. Revista Árvore, Viçosa, MG, v. 7, n. 1, p. 56-70, 1983.

PIRES, L. M.; CALEGÁRIO, N. Ajuste de modelos estocásticos lineares e não lineares para a descrição do perfil longitudinal de árvores. Revista Árvore, Viçosa, MG, v. 31, n. 5, p. 845-852, 2007.

SILVA, F.; DALLA CORTE, A. P.; SANQUETTA, C. R. Equações de afilamento para descrever o volume total do fuste de Pinus caribaea var. hondurensis na região do Triângulo Mineiro. Scientia Forestalis, Piracicaba, v. 39, n. 91, p. 367-376, 2011.

SOUZA, C. A. M.; SILVA, G. F.; XAVIER, A. C.; CHICHORRO, J. F.; SOARES, C. P. B.; SOUZA, A. L. Avaliação de modelos de afilamento segmentados na estimação da altura e volume comercial de fustes de Eucalyptus sp. Revista Árvore, Viçosa, MG, v. 32, n. 3, p. 453-463, 2008. 
